\title{
Timeliness of Service Delivery for Children with Later-identified Mild to Severe Hearing Loss
}

\author{
Elizabeth A. Walker, Ph.D. ${ }^{a}$, Lenore Holte, Ph.D. ${ }^{a, b}$, Meredith Spratford, AuD.d, Jacob \\ Oleson, Ph.D. ${ }^{C}$, Anne Welhaven, M.S. ${ }^{c}$, and Melody Harrison, Ph.D. ${ }^{e}$ \\ aDepartment of Communication Sciences and Disorders, University of lowa, lowa City, IA \\ ${ }^{b}$ Department of Pediatrics, University of lowa, lowa City, IA \\ 'Department of Biostatistics, University of lowa, lowa City, IA \\ dBoys Town National Research Hospital, Omaha, NE \\ eDepartment of Otolaryngology, University of North Carolina, Chapel Hill, NC
}

\begin{abstract}
Purpose-This study examined diagnostic and intervention services for children identified with hearing loss (HL) after the newborn period.

Method-We compared ages at service delivery and length of delays between service delivery steps for 57 later-identified children with HL and 193 children who referred for assessment from the newborn hearing screen (NHS). For only later-identified children, regression models were used to investigate relationships among predictor variables and dependent variables related to service delivery.
\end{abstract}

Results-Children who referred from the NHS received follow-up services at younger ages than later-identified children. Later-identified children had significantly longer delays between entry into early intervention to HL confirmation, compared to children who referred from the NHS. For later-identified children, degree of HL predicted ages at follow-up clinical services. Children with more severe HL received services at younger ages compared to children with milder HL. Gender predicted the length of the delay between confirmation to entry into early intervention, with females demonstrating shorter delays.

Conclusions-The current results lend support to the need for ongoing hearing monitoring programs after the neonatal period, particularly when children enter early intervention programs because of language/developmental delays.

\section{Keywords}

hearing loss; children; early intervention

\section{Introduction}

The feasibility of early identification and intervention for children with hearing loss (HL) has changed dramatically as a result of universal newborn hearing screening (UNHS)

Copyright 2013 by American Speech-Language-Hearing Association.

Corresponding author: Elizabeth A. Walker, 250 Hawkins Drive, University of Iowa, Iowa City, IA 52252, 319-335-6851, elizabethwalker@uiowa.edu.

The authors have no financial relationships relevant to this article to disclose. 
programs, which are now the standard of care throughout the United States (YoshinagaItano, Sedey, Coulter, \& Mehl, 1998). Prior to UNHS, most children with hearing loss were identified around two years of age or later (Halpin, Smith, Widen, \& Chertoff, 2010; Stein, Clark, \& Kraus, 1983; Stein, Jabaley, Spitz, Stoakley, \& McGee, 1990). Studies confirm that UNHS is effective in decreasing the ages at which children receive follow-up [i.e., HL confirmation, entry into early intervention, and hearing aid (HA) fitting] (Dalzell et al., 2000; Durieux-Smith, Fitzpatrick, \& Whittingham, 2008; Harrison, Roush, \& Wallace, 2003; Holte et al., 2012; Sininger et al., 2009; Spivak, Sokol, Auerbach, \& Gershkovich, 2009; Vohr et al., 2008). However, not every child with HL is identified at the newborn hearing screen (NHS), and not every newborn has access to NHS. Although there are many studies describing trends in the early identification process for children who referred for assessment from the NHS, there is a paucity of research describing access to follow-up services for children identified after the newborn period. Such research may lead to a better understanding of whether or not medical, speech/language, early intervention, and audiology service providers are appropriately responsive to families of later-identified children with HL, which will in turn guide management decisions for these children and their families. The goal of the present study was to describe access to, and timeliness of, service provision (i.e., diagnostic audiologic evaluations, HA fitting, and early intervention services) for children who are hard of hearing $(\mathrm{HH})$ who were identified after the newborn period and outside of the UNHS context.

\section{Reasons for later identification of childhood hearing loss}

There is evidence that a substantial number of children with permanent HL are identified after the neonatal period (Dedhia, Kitsko, Sabo, \& Chi, 2013; Hutt \& Rhodes, 2008). Watkin and Baldwin (2011) conducted a cohort study over a 10-year period in Great Britain, after initiation of UNHS. They reported that $51 \%$ of 9 -year-olds were identified with HL after the neonatal period. Bamford et al. (2007) also noted that the prevalence of permanent $\mathrm{HL}$ increased after the newborn period. There are three primary reasons why children may be identified outside the context of UNHS: 1) postnatal onset HL, 2) congenital HL missed by the NHS test (false negative), and 3) NHS not conducted. In cases of postnatal onset of HL, it appears that around 7 to $25 \%$ of all children with HL pass the NHS and are diagnosed with HL during later childhood (Dedhia et al., 2013; H. Fortnum, 2003; Weichbold, Nekahm-Heis, \& Welzl-Mueller, 2006). Some of these children will have one of 11 identified risk factors outlined by the US Joint Committee on Infant Hearing (JCIH, 2007).

These risk factor registries can be used to identify babies who should be targeted for ongoing hearing monitoring, but in practice these registries are not very effective (Beswick, Driscoll, \& Kei, 2012; Beswick, Driscoll, Kei, \& Glennon, 2012; Hutt \& Rhodes, 2008). In the second case, some children are missed by NHS, in particular those with low frequency $\mathrm{HL}$ and/or minimal/mild degrees of HL (Davis, Reeve, Hind, \& Bamford, 2001). In addition, some cases of auditory neuropathy/dys-synchrony (AND) are missed when the newborn passes a screening only on the basis of otoacoustic emissions (OAEs) (Kirkim, Serbetcioglu, Erdag, \& Ceryan, 2008). Finally, some newborns are not screened due to birth in a non-UNHS setting, illness, or family objection to screening (Christensen, Thomson, \& Letson, 2008).

\section{Reasons to investigate access to timely service provision}

Once later-identified children are diagnosed with HL, why is it important to explore their access to follow-up services? A primary reason is that delays in identification of HL can contribute to delays in language, cognitive, social, and educational skills. Numerous studies show an association between age at identification and developmental outcomes (Kennedy et al., 2006; Moeller, 2000; Yoshinaga-Itano et al., 1998). Kennedy et al. (2006) investigated 
120 children with permanent bilateral HL (mean age $=7.9$ years). Approximately half of the participants had HL confirmed by 9 months of age, while the remaining children had HL confirmed after 9 months. After controlling for severity of HL and maternal education level, Kennedy and colleagues reported that the earlier-identified children had significantly better receptive and expressive language scores compared to later-identified children. We may conclude from these findings that children who are later-identified are at risk for communication delays, because they have a period of diminished auditory input. Children who are identified through UNHS are at an advantage due to earlier audiological intervention, HA fitting, and subsequently, earlier access to auditory input. Sininger, Grimes, and Christensen (2010) identified age at HA fitting as a significant predictor of speech perception, speech production and language scores in young children with HL, which highlights the importance of this service-delivery milestone. Therefore, it is critical to document service-delivery practices for children who are identified after the newborn period, in addition to children identified at or around birth.

Another motivation for the current investigation is that identification and audiological follow-up are the first of multiple steps in ameliorating the negative effects of HL on developmental outcomes. Once a HL is confirmed, children are enrolled in early intervention services, which is also an essential milestone in the service delivery process. Although later identification can result in speech and language delays (Kennedy et al., 2006; Sininger et al., 2010), these delays may resolve after later-identified children are appropriately aided and enrolled in intervention. Moeller et al. (2010) followed four HH children who were identified at an average age of 30.2 months (range 20 to 39 months) and were provided with amplification within two months of diagnosis (mean $=1.3$ months postidentification). The children were enrolled in early intervention or preschool services for children with HL. All four children showed significant delays in speech and language skills upon entry into the study (around the time of identification of HL); however, three out of the four performed within the normal range on standardized speech and language measures by 60 months of age. It is worth noting that the child whose performance did not fall within the normal range had the greatest degree of $\mathrm{HL}$ and was an inconsistent HA user; the parents reported HA use at school but not in other settings, resulting in less access to auditory input. Moeller et al.'s findings suggest that children who are later-identified with HL can still show rapid growth in speech and language skills, once they receive appropriate amplification and intervention. Unfortunately, due to the small number of participants in Moeller et al. (2010), we cannot make any generalizations regarding when later-identified children typically receive amplification or intervention, once the presence of $\mathrm{HL}$ is established. We also cannot determine if certain service delivery steps are easier or more difficult for children who are later-identified to access, compared to children who are identified at birth.

If there are consistent delays at any point in the service delivery process (i.e., confirmation, HA fitting, and entry into intervention), there is clinical value in documenting these delays. Sininger et al. (2009) provide some insight into delays in HA fitting or entry into intervention. The investigators took advantage of variations in the start-up period for NHS programs to compare service delivery for children who referred from the NHS $(n=39)$, passed the NHS and were later identified $(n=7)$, or were not screened $(n=17)$. Children with HL who passed the NHS or were not screened showed a significantly later age at confirmation, HA fitting, and entry into intervention, compared to children who referred from the NHS. There was a trend for children who passed the NHS to have a slightly longer median delay between confirmation and HA fitting and a slightly shorter median delay between confirmation and entry into intervention, relative to the children who referred, however, these differences were not significant. With only seven children in the group who passed NHS, statistical analyses may have been underpowered. In summary, the study by Sininger et al. suggests that there were no group differences in delays between HL 
confirmation and HA fitting or entry in intervention between children identified at birth or later. Additional research is needed on this question, with the inclusion of a larger number of children identified after the newborn period.

Finally, documentation of the timing of service delivery for all children with HL is important because it allows us to identify predictors of timely follow-up. If specific factors influence when service delivery occurs, service providers may be able to proactively address these factors early in the intervention process. Holte et al. (2012) recently described a cohort of 193 children who referred from the NHS. The researchers examined variables (maternal education level as a proxy for socio-economic status, degree of HL, site of testing, gender) thought to predict timely follow-up. Only maternal education level was significantly related to age at confirmation and HA fitting, indicating that families from lower socio-economic backgrounds might need additional support after a child refers from the NHS. In contrast to studies conducted prior to UNHS (Coplan, 1987; Harrison \& Roush, 1996; Mace, Wallace, Whan, \& Stelmachowicz, 1991), Holte et al. found that degree of HL was not associated with age at confirmation and HA fitting. This suggests that a referral from UNHS promotes timely follow-up for all degrees of HL, even for children with mild HL. It is not clear which factors predict access to timely service delivery for children with $\mathrm{HL}$ who are later identified. We would expect to see an inverse relationship between degree of HL and ages at service delivery, because most of these children are identified by family members or teachers (Dedhia et al., 2013; Sininger et al., 2009). Similar to the early-identified group in Holte et al. (2012), we might also predict that maternal education level would impact delays in follow-up after identification of HL.

In summary, previous investigations demonstrated that a large proportion of children with HL are identified after the neonatal period (Bamford et al., 2007; Watkin \& Baldwin, 2011). To date, only one study has examined how quickly later-identified children receive HA fitting and entry into intervention once HL is identified (Sininger et al., 2009). Given our limited knowledge about trends in follow-up services for children with HL who are identified after the newborn period, the present study sought to describe characteristics of 57 later-identified children and identify variables that predicted access to timely follow-up. A secondary goal was to identify barriers to accessing services, as reported by parents. Specifically, this study addressed the following research questions:

1. Once HL is suspected, at what ages do later-identified children access follow-up services (i.e., first diagnostic evaluation, HL confirmation, HA fitting, and entry into early intervention) compared to children who referred from the NHS?

2. Are there differences in the length of delays between follow-up services for lateridentified children compared to children who referred from the NHS?

3. If there are delays in diagnostic evaluations or HA fitting, what are the reasons, as described by parents of later-identified children?

4. Which factors affect timely audiologic evaluation, HA fitting, and entry into early intervention for later-identified children?

\section{Method}

Participants

Holte et al. (2012) reported on 193 children who were HH and referred for assessment from the NHS. An additional 57 children who were HH (27 females) took part in the same longitudinal study (Outcomes of Children with Hearing Loss; OCHL), but were identified with HL after 1 month of age. This later-identified group is the focus of the current observational study, and will be referred to as "later-identified" throughout this paper. These 
children were later-identified for the following reasons: no NHS at birth $(n=5)$, passed

NHS and later demonstrated HL $(n=49)$, or parents reported that they were not aware of the NHS results and medical records did not indicate NHS status $(n=3)$. Based on a review of medical records and parent report, we confirmed that $25 \%$ of the children had a congenital hearing loss. Some passed the NHS due to mild degrees of HL or configuration (low frequency HL with normal hearing in the high frequencies). Two children failed the NHS on the first try but passed on the second; due to family history or parent report, we had reason to suspect that these children had congenital HL. Thirty-nine percent had post-natal onset of hearing loss, as a result of structural abnormalities (e.g., Enlarged Vestibular Aqueduct Syndrome) or acquired etiologies (e.g., ototoxicity, meningitis, assisted ventilation). We were unable to determine the onset of hearing loss for $36 \%$ of the group. In addition, $42 \%$ of the cohort presented with a progressive hearing loss, as determined by parent report during the intake interview or more than a $10 \mathrm{~dB}$ HL change in better-ear pure-tone average (PTA) over the course of the study.

To qualify for participation in the OCHL study, children presented with a permanent bilateral HL (sensorineural, mixed, and permanent conductive) with a better-ear three- or four-frequency PTA no better than $25 \mathrm{~dB}$ HL and no poorer than $75 \mathrm{~dB}$ HL. Children with significant cognitive, visual or motor impairments were excluded from participation. For all participants, at least one primary caregiver spoke English in the home. Children who used manually-coded English or American Sign Language as their primary mode of communication were excluded from the study. Table 1 shows the demographic characteristics of the 193 children who referred from the NHS (referred to as "NHS group"; Holte et al., 2012) and the 57 children in the later-identified group.

\section{Data collection}

As part of the OCHL study, children and their families participated in an initial baseline visit, followed by visits twice a year for children under age 2 years and once a year for children older than 2 years. At the initial visit, parents completed an intake interview with an examiner. Intake questions documented the ages at which the child met audiological and intervention benchmarks, including ages at 1) suspicion of HL, 2) first diagnostic audiologic evaluation, 3) HL confirmation, 4) HA fitting, and 5) entry into early intervention. The OCHL intake questionnaire did not ask about age at intervention for children who received speech/language therapy or related services after 36 months of age; therefore, only children receiving birth-to-3 early intervention services, as part of Part $C$ services of the Individuals with Disabilities Education Act (IDEA) were included in the current analysis on early intervention (36/57 children in the later-identified group; 171/193 in the NHS group). Parents provided explanations for delays between 1) suspicion of HL and first diagnostic evaluation and 2) HL confirmation and HA fitting. OCHL testing visits consisted of an audiologic assessment, including evaluation of HA function and administration of ageappropriate speech, language, and cognitive measures (for a full list of specific tests measures used in the OCHL study, see Holte et al., 2012 and Walker et al., 2013).

Audiologic assessment-An experienced pediatric audiologist completed all behavioral hearing assessments, with an assistant participating in assessments as needed. The audiologist attempted to obtain air-conduction and bone-conduction thresholds at 500, 1000, 2000 , and $4000 \mathrm{~Hz}$ at a minimum, using visual reinforcement audiometry, conditioned play audiometry, or conventional audiometry depending on the age of the child. All attempts were made to obtain ear-specific thresholds using insert earphones, supra-aural headphones, or the child's own earmolds paired with insert earphones. The audiologist obtained soundfield thresholds if the child would not tolerate the testing with earphones or headphones. If a full audiogram could not be completed, the audiologist obtained a copy of 
the child's most recent unaided audiogram from their clinical audiologist (in the majority of cases this audiogram was obtained within three months of the OCHL test visit). The betterear pure-tone average (BEPTA) was calculated for subsequent analyses.

\section{Data analysis}

The primary goal of the present study was to compare ages at service delivery and the length of delays between service delivery steps for children with HL who were later-identified to children identified by NHS (Holte et al., 2012). Based on results from the OCHL intake interview and audiological/medical records, we determined the ages at suspicion of HL, first diagnostic evaluation, HL confirmation, HA fitting, and entry into early intervention for the later-identified and NHS groups. We also determined the length of delays between first evaluation to confirmation and HL confirmation to HA fitting. Finally, we examined delays between confirmation and entry into early intervention. Early intervention was defined as state-based intervention services, provided to children ages birth to 3 years. Intervention included services from a teacher of the deaf/hard of hearing, speech-language pathologist (SLP), developmental therapist, occupational therapist and/or physical therapist. Some children in both groups began early intervention before HL confirmation (later-identified group, $n=16$; NHS group, $n=23$ ). In the later-identified group, early intervention services were initiated due to concerns about speech, language, motor, or global development. With regards to the NHS group, early intervention services were sometimes initiated due to referral on the NHS and suspicion of HL. Other children began early intervention after HL was confirmed (later-identified group, $\mathrm{n}=20$; NHS group, $\mathrm{n}=151$ ); in these cases, early intervention services were initiated because of the confirmation of HL. In order to more fully understand trends in service delivery for children who are $\mathrm{HH}$, we analyzed children in the former group (early intervention before HL confirmation) separately from children in the latter group (HL confirmation before early intervention). Independent two-sample t-tests were used to determine if there were significant differences between groups in the ages at service delivery and the length of delays between service delivery steps.

Additional statistical analysis focused on the influence of child and family variables on the access of audiological and early intervention services for the 57 children who were lateridentified with HL. We used regression models to identify the best predictors of dependent variables. The independent variables included gender, site of testing (University of Iowa, Boys Town National Research Hospital, and University of North Carolina-Chapel Hill), maternal education level, immediate family history of HL (parents and/or siblings), and degree of hearing loss (BEPTA) based on the earliest audiogram available during OCHL testing. Due to the large number of maternal education categories in the dataset, four arbitrary ordinal levels were introduced in the analysis. Table 2 displays the frequency and percentage of mothers comprising each of the four levels for maternal education. Ages at each follow-up step served as the dependent variables: 1) first diagnostic evaluation, 2) HL confirmation, 3) HA fitting, and 4) entry into early intervention. We also examined the time intervals between specific services: 1) length of delay between HL confirmation and HA fitting, and 2) length of delay between HL confirmation and entry into early intervention (including only children who had started early intervention after confirmation; see above explanation).

\section{Results}

\section{Comparisons of service delivery for NHS and later-identified children}

The first research question addressed the ages at which later-identified children received follow-up services, compared to the NHS group described in Holte et al. (2012). Table 3 displays means, medians, and standard deviations for age at suspicion of HL, first diagnostic 
evaluation, HL confirmation, HA fitting, and entry into early intervention (i.e., birth-to-3 services). Figure 1 compares the NHS and later-identified groups in terms of means, medians, and interquartile ranges for ages at service delivery. There was considerable variability in the ages at which the later-identified group received audiologic and intervention follow-up services. This variability is expected, given the differences within the later-identified group in ages at suspicion of HL.

Table 4 displays the results of t-tests to determine if there were significant differences between the NHS and later-identified groups in the ages at service delivery. The mean ages were significantly different (all $p$ s $<.001$ ) with the NHS group being younger at each of the steps. To further illustrate the differences in the average ages at service delivery, we evaluated the mean differences between the NHS and later-identified groups. There was a mean difference between groups of 24.3 months for age at first evaluation, 24.1 months for HL confirmation, 21.5 months for HA fitting, and 10 months for entry into early intervention. In other words, later-identified children were diagnosed and fit with HAs approximately two years later than the NHS group, but started early intervention 10 months later than the NHS group. This large discrepancy between age at HL confirmation/HA fitting and entry into early intervention was unanticipated. It is also noteworthy that the average and median age at entry into early intervention for children who were lateridentified $(\mathrm{n}=36$, mean $=16.8$, median $=15.5, \mathrm{SD}=9.88)$ occurred earlier than even age at suspicion of $\mathrm{HL}(\mathrm{n}=57$, mean $=24.2$, median $=23, \mathrm{SD}=16.7)$. A further examination of the data indicated that early intervention services preceded suspicion of HL because a number of children were initially enrolled in early intervention for reasons other than HL, specifically speech/language delays $(n=6)$, developmental/motor delays $(n=4)$, or prematurity $(\mathrm{n}=3)$, according to parent report.

\section{Comparisons of delays in service delivery between NHS and later-identified children with $\mathrm{HL}$}

The second research question compared delays in service delivery between the two groups. We established delays by subtracting the length of time between service delivery milestones (in months) for individual participants and then calculating the mean, median and standard deviation for the whole group. Table 3 displays values for the means, medians, and standard deviations for delays between milestones within each group. Note that the standard deviation and range values in Table 3 appear different between the groups, particularly for the delay between early intervention and HL confirmation and the delay between HL confirmation and HA fitting. To evaluate if variances were equal, we used Levene's test for equality of variances. This analysis reached statistical significance for the variance in the delay between entry into early intervention and HL confirmation $(F=7.71, p=.009)$, because the lateridentified group had larger variance than the NHS group. The analysis approached significance for the variance in the delay between HL confirmation and HA fitting ( $F=2.9$, $p=.09$ ), because the later-identified group had smaller variance than the NHS group. There were no significant differences in the variance for delays between first evaluation and confirmation or confirmation and entry into early intervention (both $p$-values > .35). To be conservative when comparing group means we used Welch's adjustment for unequal variances, when appropriate.

Results from the t-tests comparing mean delays between groups (Table 4) indicated that the only significant difference between the two groups was in the mean length of delay between entry into early intervention and HL confirmation $(p=.03)$. The later-identified group had a significantly longer delay between entry into early intervention and HL confirmation, compared to the NHS group. Closer inspection of the data revealed that 12 of the 16 children in the later-identified group were enrolled in early intervention prior to their first diagnostic 
hearing evaluation. Within this group of 12 children, the mean time between entry into early intervention and the first diagnostic evaluation was 14.17 months (median $=11.5, \mathrm{SD}=$ 13.44 , range $=2$ to 48 months), indicating that on average, these children received early intervention for over one year before receiving a hearing test.

There were no significant differences between the group means in length of delays between first evaluation and HL confirmation, HL confirmation and HA fitting, or HL confirmation and entry into early intervention (all $p \mathrm{~s}>.30$; see Table 4). The non-significant findings suggest that the mean delays between these benchmarks were consistent across groups. As previously stated, there was a marginally significant result for Levene's test of equal variances, in which the later-identified children tended to have smaller standard deviations between HL confirmation and HA fitting in comparison to the NHS group. This implies that the later-identified children received amplification within a smaller window of time, once HL was confirmed. In contrast, the children who referred on the NHS showed much greater variability in delays between HL confirmation and HA fitting. This variability can be seen in the SD and range values in Table 3.

We also compared when the two groups of children accessed specific services, to evaluate whether or not one of the groups had a larger proportion of children with lengthy delays between services (see Figure 2). To compare the later-identified and NHS groups, we based access to timely service delivery on an adaptation of the JCIH (2007) recommendations. The JCIH recommends that all infants who fail the NHS receive a comprehensive audiological evaluation by 3 months of age. For infants with a confirmed hearing loss, JCIH recommends HA fitting within one month of confirmation and enrollment in early intervention by 6 months of age. Thus, we identified the percentage of children in the NHS group who had HL confirmation by 3 months of age, HA fitting within one month of HL confirmation, and enrollment in early intervention by 6 months of age. We then adapted the JCIH recommendations for the later-identified group to determine the percentage who had $\mathrm{HL}$ confirmation within three months of the first evaluation, HA fitting within one month of HL confirmation, and early intervention within three months of HL confirmation (including only those children who were enrolled in early intervention after HL confirmation; NHS group, $\mathrm{n}$ $=151$; later-identified group, $\mathrm{n}=20$ ). A series of $\chi^{2}$ analyses, comparing percentages for each group at the three milestones (confirmation, HA fitting, early intervention) indicated there were no significant differences between groups (all $p \mathrm{~s}>.30$ ). As shown in Figure 2, the highest proportion of children in both groups to experience delays occurred between HL confirmation and HA fitting. Approximately half of the children in the early and lateridentified groups experienced more than a one-month delay between HL confirmation and HA fitting.

\section{Parental reasons for delays in service provision}

The third research question examined parents' reported reasons for delays in service provision between suspicion of HL and first evaluation and HL confirmation and HA fitting. Twenty-four parents reported a delay of 3 months or greater between suspicion and first evaluation of HL. Seventeen of those parents provided reasons for that delay (Figure 3). Seven parents (41\%) reported that they chose to wait before scheduling an evaluation. Some of these parents reported being in denial, feeling that their child had "selective hearing" or was tuning them out, or knew there was a problem but did not suspect HL because the child had passed the NHS. Six parents (35\%) reported delays due to medical issues, such as otitis media with effusion (OME), prematurity, neurologic problems, and heart conditions (two of these parents also reported undergoing repeated rescreens). Two parents reported that they were told by professionals that HL was not a concern because the child passed the NHS or 
the HL was temporary because of OME, and two parents reported having to wait for an appointment for the diagnostic evaluation.

Twenty-seven parents reported that there was a delay of two months or longer between HL confirmation and HA fitting. Twenty-one of these parents provided reasons for that delay including $12(57 \%)$ who reported that they chose not to proceed with HAs immediately after confirmation. Reasons included going through the grieving process, HAs were not recommended, or they were transferring care to audiology clinics that specialized in pediatric HA fittings or dealing with additional medical issues that prevented immediate follow-up (cancer, meningitis) (Figure 4). Three parents reported delays in receiving approval for insurance to pay for HAs, three reported delays in getting the clinic appointment to fit the HAs, and one reported a delay in obtaining medical clearance from a physician. Finally, two parents reported that they were unsure of the reason for the delay.

\section{Predictors of timeliness of service delivery}

The fourth research question was to identify which demographic variables were significant predictors of timely service delivery or of delays between service delivery steps. The independent predictor variables included gender, test site, maternal education level, immediate family history of HL, and degree of HL. After controlling for the other independent variables, only degree of HL was significantly related to the ages at first diagnostic evaluation $(\beta=-0.36, p=.003)$, HL confirmation $(\beta=-0.42, p=.001)$, and HA fitting $(\beta=-0.37, p=.011)$. As indicated by the strength and direction of the regression coefficients, as degree of HL increased, the ages at which various services occurred decreased. For the 20 children enrolled in early intervention following HL confirmation, gender was the only significant predictor of the length of the delay between those two steps ( $\beta=-3.34, p=.024$ ); female subjects had a shorter delay between HL confirmation and early intervention. None of the independent variables were significantly related to the age at entry into early intervention or the delay between HL confirmation and HA fitting.

\section{Discussion}

There are numerous reports documenting the effectiveness of UNHS in decreasing the age of identification of $\mathrm{HL}$ in children. Unfortunately, not all children with permanent $\mathrm{HL}$ are identified by the NHS (Beswick, Driscoll, \& Kei, 2012; H. M. Fortnum et al., 2001; Van Naarden, Decouflé, \& Caldwell, 1999; Weichbold et al., 2006). It is important to determine if later-identified children are indeed receiving timely service delivery because this research may inform management decisions for children with HL and their families. The purpose of the current study was to investigate follow-up services for a group of children with HL who were identified after the newborn period and outside of the UNHS context. The present findings expand our knowledge base regarding when these children receive follow-up diagnostic and intervention services and what factors predict delays in service delivery.

\section{Access to timely service delivery for children with $\mathrm{HL}$}

Our first two goals were to compare children who were later-identified to children identified by the NHS, both in regards to the ages at which children received important follow-up services and the length of delays between services. Not surprisingly, the NHS group attained all benchmark outcomes (age at first evaluation, HL confirmation, HA fitting, and entry into early intervention) at significantly younger ages than the later-identified group, which is consistent with results of previous studies (Sininger et al., 2009). These findings are further evidence of the effectiveness of the NHS identification process, and provide support for modifications in the hearing screening model currently in place across the United States to include ongoing hearing monitoring programs after the neonatal period (Dedhia et al., 2013; 
Eiserman, Hartel, et al., 2008; Eiserman, Shisler, et al., 2008). Such screening protocols, at a minimum, include two groups of children. The first group is composed of infants and toddlers with known risk factors for HL, but who pass neonatal screening. These children should be carefully monitored via periodic screenings for onset of HL until their third birthday. Children who qualify for a schedule of heightened vigilance include those with extended NICU stays (more than 5 days), who received extracorporeal membrane oxygenation (ECMO), assisted ventilation, chemotherapy, ototoxic medications, loop diuretics, or exchange transfusions due to hyperbilirubinemia, who presented with neurodegenerative disorders, congenital cytomegalovirus (CMV) infection, family history of $\mathrm{HL}$, trauma, postnatal infections associated with HL, or whose caregivers expressed concern about speech and language development (JCIH, 2007).

In the current dataset, 34 out of the 57 children (60\%) presented with risk factors outlined by JCIH (2007); 11 of the 57 presented with more than one risk factor. This leads us to the question of which health care professional is most likely to be involved in surveillance of HL for children with risk factors who pass the NHS. In many cases, parents will turn to their family physicians or pediatricians when they first begin to suspect a problem. Therefore, it is important for primary care providers to be aware of the risk factors associated with postnatal onset of hearing loss. Dedhia et al. (2013) conducted a retrospective analysis of 78 children who passed their NHS and were later identified with HL. Medical records indicated that only $12 \%$ of the children were referred to an otolaryngologist for hearing loss evaluation by their primary care doctors; most children were referred because of parental concerns $(36 \%)$ or failed school screenings (32\%). Dedhia et al. suggested that pediatricians and primary care doctors may overlook signs of HL in cases where children have passed the NHS, although they did not provide evidence to support this assertion. One other study examined the knowledge base of primary care physicians, with regards to identification of HL. Moeller, White, and Shisler (2006) surveyed 1968 primary care physicians about their practices and knowledge of UNHS. Half of the survey respondents were not well-informed regarding risk factors for delayed-onset $\mathrm{HL}$, which has implications for surveillance of children who may present with post-natal onset. Ongoing monitoring of hearing for children with risk factors will be more effective if primary care physicians are knowledgeable about the causes of permanent childhood HL. The presence of some of the biggest risks for delayed-onset HL often go unrecognized by parents; for example, $90 \%$ of congenital CMV is asymptomatic at birth (Fowler, Pass, Boppana, \& Britt, 1999; Ogawa et al., 2007; Williamson, Demmler, Percy, \& Catlin, 1992) and most genetic HL is nonsyndromic recessive (Petit, Levilliers, \& Hardelin, 2001). JCIH recommendations specify that family doctors should monitor developmental auditory skills in all children, including those who do not present with risk factors (JCIH, 2007). It is likely that there is a need for more public awareness about the prevalence and consequences of delayed-onset HL, particularly for risk factors such as CMV that are not immediately apparent.

As stated in the Introduction, identification of $\mathrm{HL}$ is the first step in the intervention process. It is also important to identify when children who are later-identified receive follow-up services, and if there are delays in delivery of those services. The JCIH Year 2000 Position Statement recommends an initial audiological evaluation by 3 months of age, HA fitting within one month of HL confirmation, and entry into early intervention by 6 months of age. If age at suspicion is considered the baseline, later-identified children, on average, are receiving the first diagnostic evaluation and HA fitting just slightly later than recommended. Statistical analyses revealed that there were no significant differences between the lateridentified and NHS groups, in terms of delays between first evaluation to HL confirmation and HL confirmation to HA fitting. The later-identified group tended to have smaller variance in the delay between HL confirmation and HA fitting than the NHS group, suggesting that there may be a greater sense of urgency to amplify later-identified children. 
Alternatively, the later-identified group was older than the NHS group, on average, so fewer audiologic visits may be have been required to obtain accurate behavioral thresholds and fit HAs for these children. Later-identified children who have their HL confirmed and then enter early intervention do so within the recommended timeframe. There is no significant difference in the delay between HL confirmation and entry into early intervention between the later-identified and NHS groups, suggesting that both groups are starting the early intervention process soon after HL confirmation.

We also examined the children from the present dataset who were enrolled in early intervention prior to first evaluation/HL confirmation. Our results showed that 12 children were enrolled in early intervention, also known as Part $\mathrm{C}$ services of IDEA, prior to having their hearing tested. On average, the length of the delay between entry into early intervention and the first hearing test was one year. The median delay was 5.5 months. All of these children were initially referred for services due to speech/language or developmental delays. Children who are referred to Part C programs because of speech/ language or developmental delays constitute the second group who should be included in a modified hearing screening model. We speculate that some of these children could have been identified with hearing loss earlier, if they had been screened for hearing loss when they were initially referred for Part C services. It is feasible to effectively screen for hearing loss in toddlers and preschoolers after the neonatal period, by relying on otoacoustic emissions (OAE) technology in combination with a multistep protocol (Eiserman, Hartel, et al., 2008). Studies involving children in Head Start programs demonstrate the feasibility of screening young children using such a protocol (Eiserman et al., 2007). Our recommendation is that similar protocols be implemented in other early childhood programs, such as individual states' Part C programs, in order to detect childhood hearing loss earlier. Currently, it is not standard practice for children enrolled in Part C programs for reasons other than hearing loss to have regular hearing tests (White, Forsman, Eichwald, \& Munoz, 2010). In cases where children refer for speech and language delays, it is the responsibility of the SLP to ensure that children are screened for hearing loss as part of the initial evaluation process. In other cases where children refer for more global developmental delays, it is the responsibility of the early childhood service coordinator to ensure that hearing loss is ruled out as a causal factor for delays. Regardless of which service provider initiates the hearing screen, identifying hearing loss as the cause of speech and language delays will lead to more appropriate clinical management for these children.

We can compare the current results with past studies, in order to evaluate how service delivery has changed (or stayed the same) over time. In the current analysis, we established delays between service delivery steps by subtracting the delay between steps (in months) for individual participants and then calculating measures of central tendency for the whole group, to allow for statistical comparison between groups. Previous studies used a different approach for determining length of delays, by subtracting the difference in median values. If we use the latter metric, there is a median delay of 7 months between suspicion of $\mathrm{HL}$ and HL confirmation for the later-identified children. This finding is equivalent to results reported by Harrison and Roush (1996) for preschool-aged children, prior to the advent of UNHS. The current results also indicate that the delay between HL confirmation and HA fitting continues to decrease with time, not just for children in the NHS group, but also in the later-identified group. In 1996, Harrison and Roush reported a 6 month delay between HL diagnosis and HA fitting. Seven years later, when Harrison, Roush and Wallace (2003) published a follow-up study, the delay between confirmation and HA fitting was still 5.5 months for those children whose hearing was not screened at birth and for whom no risk factors for HL were present. The current results indicate a median 2-week delay between confirmation and HA fitting for later-identified children. Sininger et al. (2009) is the only other study to describe service delivery trends for later-identified children who were born 
during or after the transition to UNHS. They reported a median delay of 1.58 and 1.94 months for later-identified children who passed the NHS, or were not screened, respectively. Taken together, the results of the present study and Sininger et al. clearly demonstrate a growing awareness of the importance of early amplification in the field of pediatric audiology and otolaryngology.

We must also consider, however, that the current results show a large proportion of children not meeting the JCIH (2007) recommendations for HA fittings within one month of confirmation. This was true for both groups: approximately half of the children in the lateridentified group and half of the children in the NHS group experienced delays in HA fitting, based on the JCIH recommendations. The results of the parent interviews point to several areas that can be addressed to decrease the delay between HL confirmation and HA fitting. Some reasons for delays in the fitting process are outside of the control of professionals, such as additional medical issues that require more immediate attention. Other areas of delay, however, may be more amenable to change, such as reducing the delay in obtaining clinic appointments, receiving medical clearance from physicians, and receiving approval from insurance. These same barriers were previously identified by Harrison and Roush (1996) and Harrison, Roush, and Wallace (2003), indicating that families are encountering the same problems with access to amplification that they were over 15 years ago.

Addressing these areas of delay requires a multidisciplinary team approach, in which audiologists, physicians, and insurance carriers are all cognizant of the importance of early, consistent access to auditory input through the use of HAs. Finally, some parents reported delaying the HA fitting because they were grieving. It should be recognized that parents of later-identified children with HL may experience protracted guilt or even denial because of the circumstances surrounding the identification of HL. Professionals may need to provide additional support in these situations, such as recommending the family attend a parent support group or outside counseling, to help them cope with their child's diagnosis.

\section{Predictors of timeliness of service delivery}

Another goal of this study was to determine if there were significant predictors for timely service delivery. In the NHS group, maternal education level was the only variable that was associated with timeliness of HL confirmation or HA fitting; children with earlier ages of HL confirmation or HA fitting tended to have mothers with higher levels of maternal education (Holte et al., 2012). In contrast, the only significant predictor of timely follow-up in the later-identified group was degree of HL. Children with more severe HL tended to have earlier ages at first evaluation, HL confirmation, and HA fitting. These results are consistent with studies prior to the advent of UNHS, which showed an inverse relationship between HL severity and timeliness of follow-up (Coplan, 1987; Harrison \& Roush, 1996; Mace et al., 1991). More recently, Sininger et al. (2009) found a negative relationship between severity of HL and age at diagnosis of HL for children who either passed the NHS or were not screened during the newborn period. Consistent with Holte et al. (2012), Sininger and colleagues did not find a relationship between degree of HL and age at diagnosis for children who referred on the NHS. Taken together, these findings lend additional support to our recommendation that standardized hearing screening protocols should be an integral component of early childhood special education programs. If all children who enter early intervention are screened for HL at entry, it may be possible to identify more children with mild to moderate HL. The influence of degree of HL also brings up the question of whether Early Hearing Detection and Intervention (EHDI) programs should implement additional screens after the newborn period. Based on the results of the parent interviews, it appears that many parents chose to wait before scheduling a hearing test, even though they suspected there was a problem with their child's development. As Dedhia et al. (2013) also proposed, some parents may experience a false sense of security 
regarding hearing status, because their child passed the NHS. A second-stage screening process may help to identify HL in those children whose parents are aware of delays in speech and language development, but do not attribute those delays to HL and therefore do not request an audiologic evaluation. It is important to note that evidence-based research on the cost effectiveness of second-stage screening is needed before implementation of a second stage could be considered (Dedhia et al., 2013).

It was also surprising that gender was the only significant predictor in the length of delay between HL confirmation and enrollment in early intervention. Girls tended to have a shorter delay between HL confirmation and entry into early intervention than boys. We speculate that there are higher expectations for communication development in young girls when they are compared with their normal-hearing female peers, particularly with regards to speech production. Gender accounts for a very small proportion of variance in early language skills (Fenson et al., 1994), however, a meta-analysis by Hyde and Linn (1988) indicated that 10 to $15 \%$ of variance in speech production can be accounted for by gender. Perhaps speech delays in girls are more noticeable during toddlerhood; thus, family members and service providers are more proactive about initiating early intervention services for girls than boys. We also anticipate that communication delays in male toddlers may be more easily dismissed as behavioral issues. There may be a need to increase efforts to ensure that children of both genders are receiving timely and appropriate intervention services once hearing loss is confirmed.

\section{Future directions and limitations}

This study reports on the ages of identification, HL confirmation, HA fitting and entry into early intervention for a carefully described cohort of children who were identified after the neonatal period and were enrolled in a five-year longitudinal research study regarding outcomes of children with mild-severe hearing loss. These children all had bilateral HL without other significant co-morbid conditions. All lived in households where at least one parent spoke English fluently. As is typical in most longitudinal studies, family income and levels of education were slightly skewed in the direction of higher incomes and more highly educated than the general population. Among children with co-occurring disabilities, HL may be masked for a longer period of time by the complex developmental challenges they present. Thus, the current findings present a best-case scenario in regards to the ages at which children with HL receive audiological and intervention services. A population-based study representing the full spectrum of children with HL needs to be conducted to more accurately determine when later-identified children gain access to services that support access to audition. Advances made in the past 15 years in identification of $\mathrm{HL}$ in newborns are remarkable; however, there is growing evidence that NHS is not a complete solution to identification of HL in all children. This report provides evidence to support careful consideration of establishment of systematic re-screening for at least two groups of children: 1) those with identified risk factors for the development of HL, and 2) those who qualify for early intervention (Part C) services.

An additional limitation of the current study is that we relied on parent report for explanations of delays in service delivery. This same procedure was utilized in an earlier publication (Holte et al., 2012). In some cases, several years had elapsed between the service delivery time points and the intake interview for the longitudinal study; therefore, it is possible that parents' memories of why there were delays could be vague. We attempted to reduce opportunities for errors by presenting parents with forced-choice alternatives for delays, rather than asking open-ended questions. Nevertheless, it is acknowledged that the parent report data were collected retrospectively and we did not have any means for verifying parents' recollections, particularly subjective reasons such as denial. Thus, results related to explanations of delays in service delivery should be interpreted with some caution. 
It may be necessary to conduct future research that prospectively follows children at the point of HL confirmation, in order to accurately identify causes of delays during the service delivery process.

In conclusion, the present study found the following results:

1. For children who are HH who are identified outside the context of NHS, the average delay between suspicion and confirmation of $\mathrm{HL}$ was 6.73 months and the average delay between HL confirmation and HA fitting was 2.6 months. The average and median age at entry into early intervention occurred earlier than suspicion of HL.

2. There were no significant differences in delays between service delivery steps for children in the NHS group and children in the later-identified group, with the exception of delay between entry into early intervention to HL confirmation. Compared to those children in the NHS group who began intervention before confirmation of HL, children in the later-identified group had a significantly longer delay and greater variance between the start of early intervention and HL confirmation.

3. Most families who experienced extended delays between suspicion of $\mathrm{HL}$ and first diagnostic evaluation, and HL confirmation and HA fitting, attributed those delays to their own decisions to postpone service delivery.

4. Degree of HL was the only significant predictor of ages at first diagnostic evaluation, HL confirmation, and HA fitting. As degree of HL increased, the ages at service delivery benchmarks decreased. Gender was the only significant predictor of the length of delay between HL confirmation and entry into early intervention; girls had a shorter delay than boys.

\section{Acknowledgments}

This work was supported by National Institutes of Health Grants NIH/NIDCD 5 ROI DC009560-03 (co-principal investigators, J. Bruce Tomblin, University of Iowa and Mary Pat Moeller, Boys Town National Research Hospital). The content of this project is solely the responsibility of the authors and does not necessarily represent the official views of the National Institute on Deafness and Other Communication Disorders or the National Institutes of Health. The authors thank Mary Pat Moeller, Ryan McCreery, Brittan Barker, and Derek Stiles for their helpful comments on earlier versions of this paper.

\section{References}

Bamford J, Fortnum H, Bristow K, Smith J, Vamvakas G, Davies L, Davis A. Current practice, accuracy, effectiveness and cost-effectiveness of the school entry hearing screen. Health Technology Assessment. 2007; 11(32):1-188. [PubMed: 17683682]

Beswick R, Driscoll C, Kei J. Monitoring for postnatal hearing loss using risk factors: a systematic literature review. Ear and Hearing. 2012; 33(6):745-756. [PubMed: 22955247]

Beswick R, Driscoll C, Kei J, Glennon S. Targeted surveillance for postnatal hearing loss: A program evaluation. International Journal of Pediatric Otorhinolaryngology. 2012; 76(7):1046-1056. [PubMed: 22560230]

Christensen M, Thomson V, Letson GW. Evaluating the reach of universal newborn hearing screening in Colorado. American Journal of Preventive Medicine. 2008; 35(6):594-597. [PubMed: 19000848]

Coplan J. Deafness: ever heard of it? Delayed recognition of permanent hearing loss. Pediatrics. 1987; 79(2):206-213. [PubMed: 2433675]

Dalzell L, Orlando M, MacDonald M, Berg A, Bradley M, Cacace A, Greenberg E. The New York State universal newborn hearing screening demonstration project: ages of hearing loss identification, hearing aid fitting, and enrollment in early intervention. Ear and Hearing. 2000; 21(2):118-130. [PubMed: 10777019] 
Davis A, Reeve K, Hind S, Bamford J. Children with mild and unilateral hearing impairment. A sound foundation through early amplification. 2001; 4:179-186.

Dedhia K, Kitsko D, Sabo D, Chi DH. Children With Sensorineural Hearing Loss After Passing the Newborn Hearing ScreenHearing Loss After Passing Newborn Hearing Screen. JAMA Otolaryngology-Head \& Neck Surgery. 2013; 139(2):119-123. [PubMed: 23328914]

Durieux-Smith A, Fitzpatrick E, Whittingham J. Universal newborn hearing screening: A question of evidence. International Journal of Audiology. 2008; 47(1):1-10. [PubMed: 18196481]

Eiserman WD, Hartel DM, Shisler L, Buhrmann J, White KR, Foust T. Using otoacoustic emissions to screen for hearing loss in early childhood care settings. International Journal of Pediatric Otorhinolaryngology. 2008; 72(4):475-482. [PubMed: 18276019]

Eiserman WD, Shisler L, Foust T, Buhrmann J, Winston R, White K. Updating hearing screening practices in early childhood settings. Infants \& Young Children. 2008; 21(3):186-193.

Eiserman WD, Shisler L, Foust T, Buhrmann J, Winston R, White KR. Screening for hearing loss in early childhood programs. Early Childhood Research Quarterly. 2007; 22(1):105-117.

Fenson L, Dale PS, Reznick JS, Bates E, Thal DJ, Pethick SJ, Stiles J. Variability in early communicative development. Monographs of the Society for Research in Child Development. 1994:i-185.

Fortnum H. Epidemiology of permanent childhood hearing impairment: implications for neonatal hearing screening. Audiological Medicine. 2003; 1(3):155-164.

Fortnum HM, Summerfield AQ, Marshall DH, Davis AC, Bamford JM, Davis A, Hind S. Prevalence of permanent childhood hearing impairment in the United Kingdom and implications for universal neonatal hearing screening: questionnaire based ascertainment study Commentary: Universal newborn hearing screening: implications for coordinating and developing services for deaf and hearing impaired children. British Medical Journal. 2001; 323(7312):536-539. [PubMed: 11546698]

Fowler KB, Pass RF, Boppana SB, Britt WJ. Neurodevelopmental Deficits in School-Aged Children Who Have Asymptomatic Congenital Cytomegalovirus (CMV) Infection. Pediatric Research. 1999; 45:161A-161A.

Halpin KS, Smith KY, Widen JE, Chertoff ME. Effects of universal newborn hearing screening on an early intervention program for children with hearing loss, birth to $3 \mathrm{yr}$ of age. Journal of the American Academy of Audiology. 2010; 21(3):169-175. [PubMed: 20211121]

Harrison M, Roush J. Age of suspicion, identification, and intervention for infants and young children with hearing loss: a national study. Ear and Hearing. 1996; 17(1):55-62. [PubMed: 8741968]

Harrison M, Roush J, Wallace J. Trends in age of identification and intervention in infants with hearing loss. Ear and Hearing. 2003; 24(1):89-95. [PubMed: 12598815]

Joint Committee on Infant Hearing. Year 2007 position statement: principles and guidelines for early hearing detection and intervention programs. Pediatrics. 120(4):898-921. [PubMed: 17908777]

Holte L, Walker E, Oleson J, Spratford M, Moeller MP, Roush P, Tomblin JB. Factors Influencing Follow-Up to Newborn Hearing Screening for Infants Who Are Hard of Hearing. American Journal of Audiology. 2012; 21(2):163-174. [PubMed: 22585937]

Hutt N, Rhodes C. Post-natal hearing loss in universal neonatal hearing screening communities: Current limitations and future directions. Journal of Paediatrics and Child Health. 2008; 44(3):8791. [PubMed: 18307417]

Hyde JS, Linn MC. Gender differences in verbal ability: A meta-analysis. Psychological Bulletin. 1988; 104(1):53-69.

Kennedy CR, McCann DC, Campbell MJ, Law CM, Mullee M, Petrou S, Stevenson J. Language ability after early detection of permanent childhood hearing impairment. New England Journal of Medicine. 2006; 354(20):2131-2141. [PubMed: 16707750]

Kirkim G, Serbetcioglu B, Erdag TK, Ceryan K. The frequency of auditory neuropathy detected by universal newborn hearing screening program. International Journal of Pediatric Otorhinolaryngology. 2008; 72(10):1461-1469. [PubMed: 18674822]

Mace AL, Wallace KL, Whan MQ, Stelmachowicz PG. Relevant factors in the identification of hearing loss. Ear and Hearing. 1991; 12(4):287-293. [PubMed: 1723705] 
Moeller MP. Early intervention and language development in children who are deaf and hard of hearing. Pediatrics. 2000; 106(3):e43-e43. [PubMed: 10969127]

Moeller MP, McCleary E, Putman C, Tyler-Krings A, Hoover B, Stelmachowicz P. Longitudinal development of phonology and morphology in children with late-identified mild-moderate sensorineural hearing loss. Ear and Hearing. 2010; 31(5):625-635. [PubMed: 20548239]

Moeller MP, White KR, Shisler L. Primary care physicians' knowledge, attitudes, and practices related to newborn hearing screening. Pediatrics. 2006; 118(4):1357-1370. [PubMed: 17015524]

Ogawa H, Suzutan T, Baba Y, Koyano S, Nozawa N, Ishibashi K, Omori K. Etiology of severe sensorineural hearing loss in children: independent impact of congenital cytomegalovirus infection and GJB2 mutations. Journal of Infectious Diseases. 2007; 195(6):782-788. [PubMed: 17299707]

Petit C, Levilliers J, Hardelin JP. Molecular genetics of hearing loss. Annual Review of Genetics. 2001; 35(1):589-645.

Sininger YS, Grimes A, Christensen E. Auditory development in early amplified children: factors influencing auditory-based communication outcomes in children with hearing loss. Ear and Hearing. 2010; 31(2):166-185. [PubMed: 20081537]

Sininger YS, Martinez A, Eisenberg L, Christensen E, Grimes A, Hu J. Newborn hearing screening speeds diagnosis and access to intervention by 20-25 months. Journal of the American Academy of Audiology. 2009; 20(1):49-57. [PubMed: 19927682]

Spivak L, Sokol H, Auerbach C, Gershkovich S. Newborn hearing screening follow-up: factors affecting hearing aid fitting by 6 months of age. American Journal of Audiology. 2009; 18(1):2433. [PubMed: 19029532]

Stein L, Clark S, Kraus N. The hearing-impaired infant: patterns of identification and habilitation. Ear and Hearing. 1983; 4(5):232-236. [PubMed: 6628846]

Stein L, Jabaley T, Spitz R, Stoakley D, McGee T. The hearing-impaired infant: patterns of identification and habilitation revisited. Ear and Hearing. 1990; 11(3):201-205. [PubMed: 2358130]

Van Naarden K, Decouflé P, Caldwell K. Prevalence and characteristics of children with serious hearing impairment in metropolitan Atlanta, 1991-1993. Pediatrics. 1999; 103(3):570-575. [PubMed: 10049958]

Vohr B, Jodoin-Krauzyk J, Tucker R, Johnson MJ, Topol D, Ahlgren M. Early language outcomes of early-identified infants with permanent hearing loss at 12 to 16 months of age. Pediatrics. 2008; 122(3):535-544. [PubMed: 18762523]

Walker EA, Spratford M, Moeller MP, Oleson J, Ou H, Roush P, Jacobs S. Predictors of Hearing Aid Use Time in Children With Mild-to-Severe Hearing Loss. Language, Speech, and Hearing Services in Schools. 2013; 44(1):73-88.

Watkin P, Baldwin M. Identifying deafness in early childhood: requirements after the newborn hearing screen. Archives of Disease in Childhood. 2011; 96(1):62-66. [PubMed: 21047829]

Weichbold V, Nekahm-Heis D, Welzl-Mueller K. Universal newborn hearing screening and postnatal hearing loss. Pediatrics. 2006; 117(4):e631-e636. [PubMed: 16585279]

White, KR.; Forsman, I.; Eichwald, J.; Munoz, K. The evolution of early hearing detection and intervention programs in the United States; Paper presented at the Seminars in perinatology; 2010.

Williamson WD, Demmler GJ, Percy AK, Catlin FI. Progressive hearing loss in infants with asymptomatic congenital cytomegalovirus infection. Pediatrics. 1992; 90(6):862-866. [PubMed: 1331946]

Yoshinaga-Itano C, Sedey AL, Coulter DK, Mehl AL. Language of early-and later-identified children with hearing loss. Pediatrics. 1998; 102(5):1161-1171. [PubMed: 9794949] 


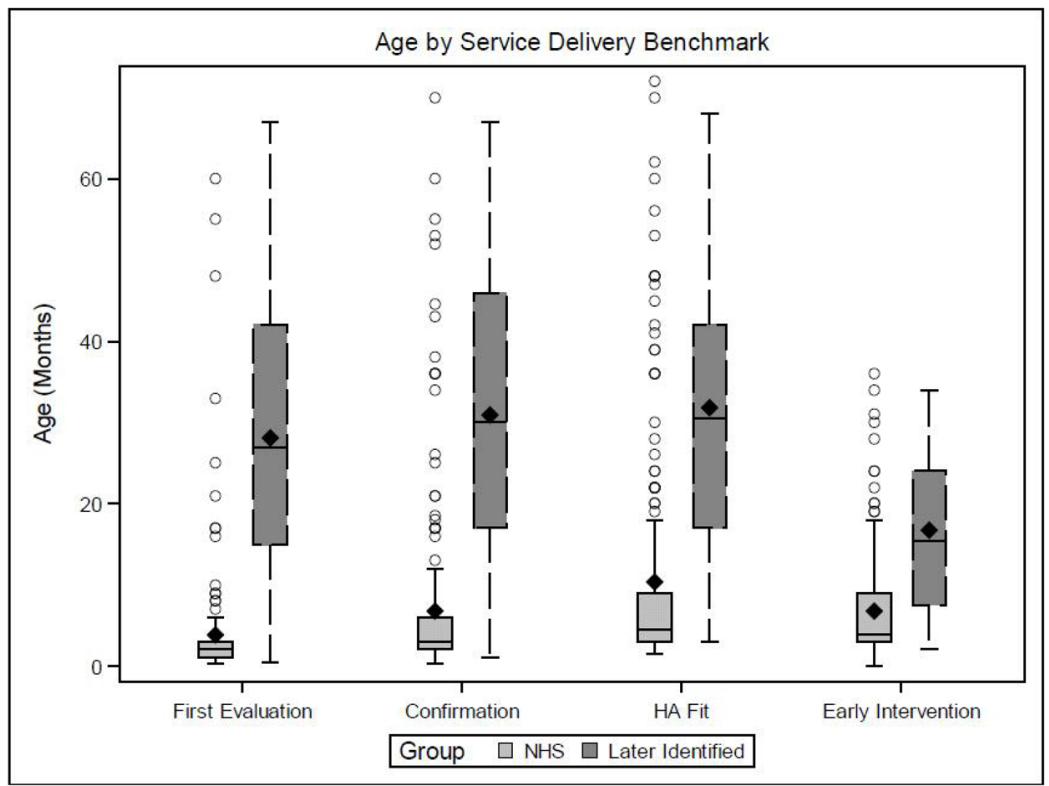

Figure 1.

Box plot of the ages at service delivery for children who referred from the NHS and children who were later identified. The central line represents the median age for each group, the diamond represents the mean age for each group, the box limits are the $25^{\text {th }}$ and $75^{\text {th }}$ percentiles, and the circles represent outliers. The lower fence is $1.5^{*}(\mathrm{IQR})$ below the $25^{\text {th }}$ percentile and the upper fence is $1.5^{*}(\mathrm{IQR})$ above the $75^{\text {th }}$ percentile (IQR $=75^{\text {th }}$ percentile $-25^{\text {th }}$ percentile). 


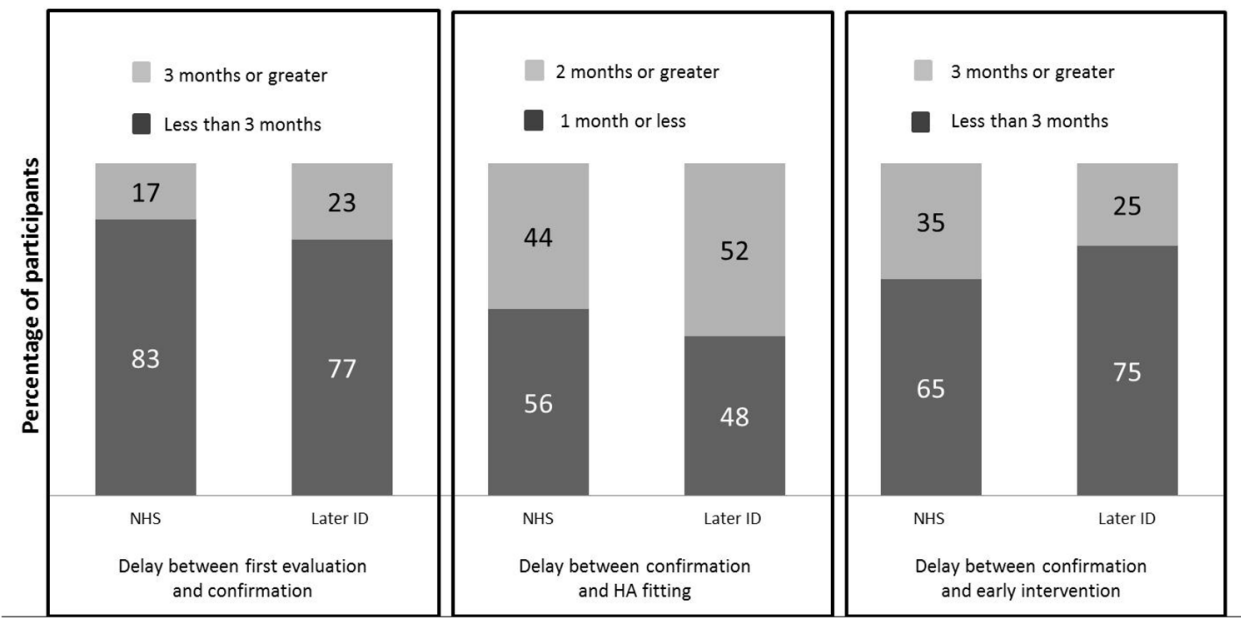

Figure 2.

Percentage of later-identified and NHS groups who experienced acceptable delays in service delivery (dark bars) or longer delays in service delivery (light bars), based on an adaptation of JCIH (2007) recommendations. 

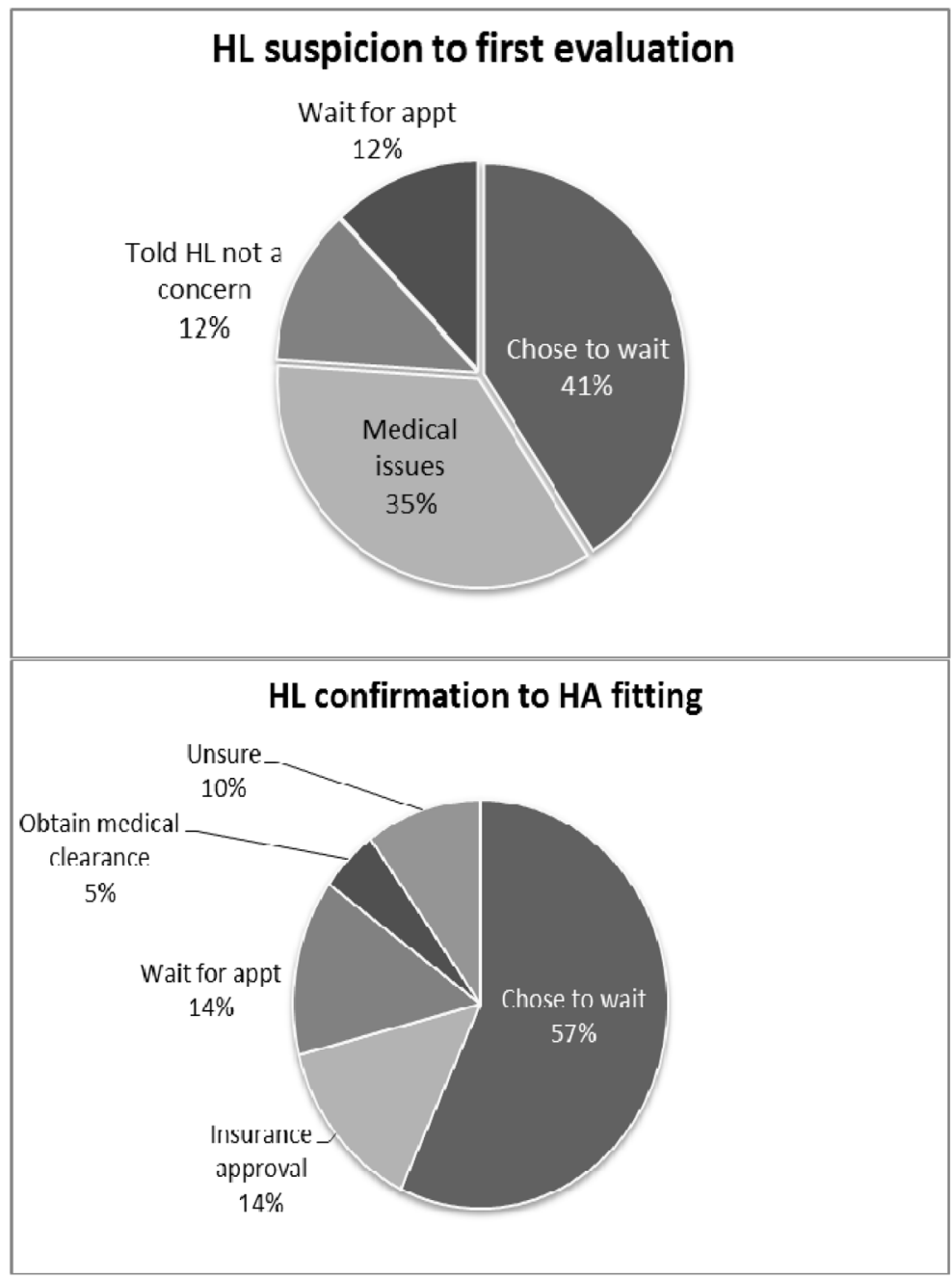

Figure 3.

Parent-report reasons for delays between suspicion of HL to first evaluation (top panel) and HL confirmation to HA fitting (bottom panel). 


\section{Table 1}

Demographic characteristics of OCHL participants who were referred on the NHS (Holte et al., 2012) or were later-identified (current study).

\begin{tabular}{|c|c|c|c|c|}
\hline \multirow[b]{2}{*}{ Demographics characteristics } & \multicolumn{2}{|c|}{ Identified by NHS ( $n=193$ ) } & \multicolumn{2}{|c|}{ Later-Identified $(n=57)$} \\
\hline & $\mathbf{n}$ & $\%$ & $\mathbf{n}$ & $\%$ \\
\hline \multicolumn{5}{|l|}{ Gender } \\
\hline Male & 103 & 53.4 & 30 & 52.6 \\
\hline Female & 90 & 46.6 & 27 & 47.4 \\
\hline \multicolumn{5}{|l|}{ Maternal education level } \\
\hline High school or less & 34 & 18 & 3 & 5 \\
\hline Some college & 65 & 39 & 17 & 30 \\
\hline Bachelor's degree & 50 & 26 & 15 & 26 \\
\hline Post graduate & 43 & 22 & 22 & 39 \\
\hline \multicolumn{5}{|l|}{ Self-identified race } \\
\hline White & 151 & 78.2 & 47 & 82.5 \\
\hline Black & 12 & 6.2 & 3 & 5.3 \\
\hline Hispanic & 5 & 2.6 & 3 & 5.3 \\
\hline Asian & 4 & 2.1 & 1 & 1.8 \\
\hline Multi-racial & 11 & 5.7 & 3 & 5.3 \\
\hline Other & 10 & 5.2 & 0 & 0 \\
\hline \multicolumn{5}{|l|}{ Research site } \\
\hline Iowa & 57 & 30 & 20 & 35 \\
\hline Nebraska & 68 & 35 & 17 & 30 \\
\hline North Carolina & 68 & 35 & 20 & 35 \\
\hline $\mathrm{JCIH}$ risk factors (one or more) & $\mathrm{n} / \mathrm{a}$ & $\mathrm{n} / \mathrm{a}$ & 34 & 60 \\
\hline
\end{tabular}


Table 2

Arbitrary levels for maternal education $(N=57)$

\begin{tabular}{l|c|c}
\hline Maternal education level & $\boldsymbol{N}$ & percentage \\
\hline High school or less & 3 & 5.26 \\
Vocational school or some college & 17 & 29.82 \\
College & 15 & 26.32 \\
Graduate school & 22 & 38.60 \\
\hline
\end{tabular}




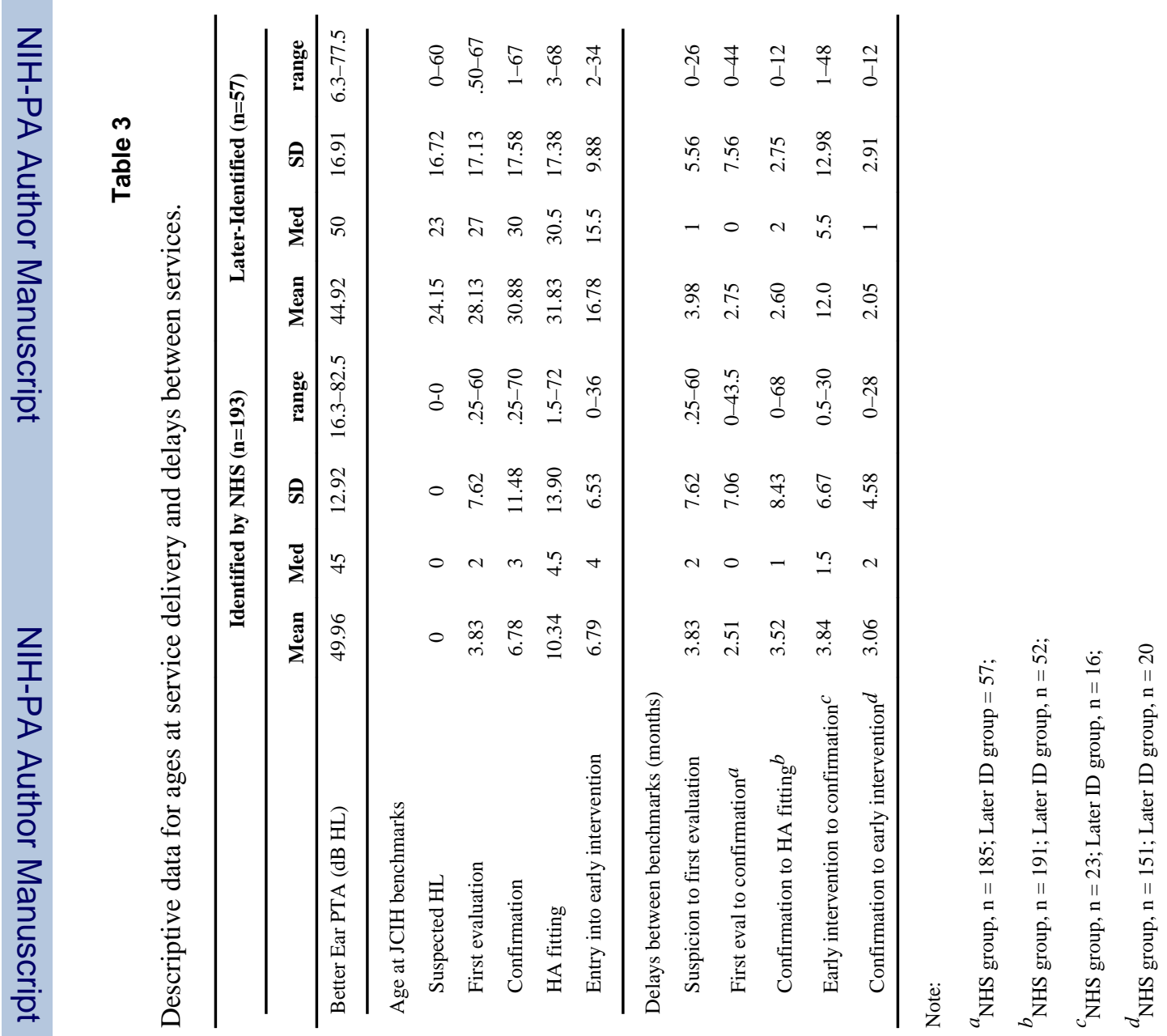


Table 4

Results of t-tests comparing NHS and later-identified groups in terms of ages at service delivery and delays between services.

\begin{tabular}{|c|c|c|c|c|}
\hline & $d f$ & $t$ & $p$ & Mean difference (in months) \\
\hline \multicolumn{5}{|l|}{ Age at JCIH benchmarks } \\
\hline First evaluation $^{a}$ & 62.93 & -10.40 & $<0.001$ & 24.31 \\
\hline Confirmation $^{a}$ & 70.67 & -9.75 & $<0.001$ & 24.10 \\
\hline HA fitting $a$ & 69.54 & -8.23 & $<0.001$ & 21.49 \\
\hline Entry into early intervention $a$ & 41.66 & -5.80 & $<0.001$ & 9.98 \\
\hline \multicolumn{5}{|l|}{ Delays between benchmarks } \\
\hline First evaluation to confirmation & 240 & -.21 & .83 & .23 \\
\hline Confirmation to HA fitting & 241 & .79 & .43 & .93 \\
\hline Early intervention to confirmation $a$ & 20.54 & 2.31 & .03 & 8.15 \\
\hline Confirmation to early intervention & 166 & .959 & .34 & 1.01 \\
\hline
\end{tabular}

Note:

${ }^{a}$ Tests of homogeneity of variance indicated that there were significant differences in the variance between groups. Degrees of freedom, t-test values, and p-values are presented using Welch's adjustment when equal variances cannot be assumed. 